\title{
Energy spectra of finite temperature superfluid helium-4 turbulence
}

\author{
Demosthenes Kivotides \\ Department of Aeronautics, Imperial College London, London SW7 2AZ, United Kingdom
}

(Received 22 June 2014; accepted 7 October 2014; published online 22 October 2014)

\begin{abstract}
A mesoscopic model of finite temperature superfluid helium-4 based on coupled Langevin-Navier-Stokes dynamics is proposed. Drawing upon scaling arguments and available numerical results, a numerical method for designing well resolved, mesoscopic calculations of finite temperature superfluid turbulence is developed. The application of model and numerical method to the problem of fully developed turbulence decay in helium II, indicates that the spectral structure of normal-fluid and superfluid turbulence is significantly more complex than that of turbulence in simplefluids. Analysis based on a forced flow of helium- 4 at $1.3 \mathrm{~K}$, where viscous dissipation in the normal-fluid is compensated by the Lundgren force, indicate three scaling regimes in the normal-fluid, that include the inertial, low wavenumber, Kolmogorov $k^{-5 / 3}$ regime, a sub-turbulence, low Reynolds number, fluctuating $k^{-2.2}$ regime, and an intermediate, viscous $k^{-6}$ range that connects the two. The $k^{-2.2}$ regime is due to normal-fluid forcing by superfluid vortices at high wavenumbers. There are also three scaling regimes in the superfluid, that include a $k^{-3}$ range that corresponds to the growth of superfluid vortex instabilities due to mutual-friction action, and an adjacent, low wavenumber, $k^{-5 / 3}$ regime that emerges during the termination of this growth, as superfluid vortices agglomerate between intense normal-fluid vorticity regions, and weakly polarized bundles are formed. There is also evidence of a high wavenumber $k^{-1}$ range that corresponds to the probing of individual-vortex velocity fields. The Kelvin waves cascade (the main dynamical effect in zero temperature superfluids) appears to be damped at the intervortex space scale. (c) 2014 AIP Publishing LLC. [http://dx.doi.org/10.1063/1.4898666]
\end{abstract}

\section{INTRODUCTION}

In analogy with classical particle systems, the physics of quantum particles also include a hydrodynamic range of scales. In many important cases, e.g., quark-gluon plasma, the hydrodynamics of quantum systems are similar to classical, ${ }^{1}$ hence, they do not pose any new fundamental problems for fluid dynamics. A very important exception are systems undergoing a Bose-Einstein condensation which is responsible for the phenomenon of superfluidity, and gives rise to the two-fluid model of Landau and Tisza. Indeed, below a critical temperature, superfluidity is responsible for the emergence of an inviscid fluid ("superfluid") whose dynamics need to be considered in conjunction with the dynamics of a standard simple-fluid, called for this reason "normal-fluid," in order to model real-life helium II experiments. Although the superfluid is inviscid, it is capable of supporting vortical modes of flow via the appearance of topological defects, also known as, quantized vortices. A complicated tangle of discrete, quantized vortices is refered to as "superfluid turbulence," ${ }^{,-7}$ and its hydrodynamic description made necessary an extension of the Landau-Tisza model. Notable such extensions are the Hall-Vinen and Gorter-Mellink equations of two-fluid hydrodynamics with vortices. In agreement with their hydrodynamic nature, these equations refer to a continuum distribution of superfluid vorticity.

An important development in superfluid turbulence studies was the vortex dynamical reformulation of the Hall-Vinen equations by Schwarz. ${ }^{8}$ The great advantage of Schwarz's approach is an 
explicit understanding of superfluid vorticity on par with similar, simple-fluid dynamics studies, for a wide range of temperatures, $T \geq 1 \mathrm{~K}$. Its limitations include a lack of a self-consistent evolution of the normal-fluid (which follows Hall-Vinen dynamics) and, by default, its macroscopic character. On the other side of the research spectrum, there are microscopic studies of superfluid turbulence based on the Gross-Pitaevskii equation. ${ }^{9-11}$ Their great advantage is their inclusion of complete quantized vortex physics, such as compressibility and vortex core dynamics during reconnections. But there are great limitations too: Gross-Pitaevskii physics only apply to weakly interacting, Bose-Einstein condensed, dilute gases, hence, they offer, at most, a qualitative understanding of strongly interacting helium II physics. Moreover, they do not include finite temperature effects, and even close to absolute zero, they only model the condensate atoms and not the totality of superfluid atoms.

In between these limiting cases, there is the mesoscopic regime of superfluid dynamics that includes discrete (rather than continuous) superfluid vortices interacting with a continuous, NavierStokes normal-fluid. ${ }^{1}$ The present research refers to this range of scales, and takes advantage of some good features, i.e., the ability to: (a) perform self-consistent computations, coupling the superfluid vortices with the normal-fluid at finite temperatures, (b) compute the explicit dynamics of individual quantized vortices, (c) inform macroscopic studies by averaging the mesoscopic variables, and (d) provide an effective description of microscopic physics of systems, that are too large for an analysis in terms of Gross-Pitaevskii or quantum field theoretic equations. The limitations of the method include its crude model of vortex reconnections, and its demanding computational complexity, that restricts its application to relatively small systems as compared with the macroscopic (Hall-Vinen, Gorter-Mellink) approach.

I propose a new, powerful, mesoscopic model of superfluid vortex dynamics, which is based on the Langevin equation, and is coupled with Navier-Stokes dynamics for the normal-fluid. I discuss at great length how to set up well resolved fully developed turbulence calculations with this model, and perform such computations for decaying, finite temperature, superfluid turbulence. Due to the lack of any prior theoretical computations of (fully coupled, mesoscopic) homogeneous, isotropic, superfluid turbulence, I concentrate here on the most important physical aspects. These are the evolution of global quantities such as energies and vortex-tangle length, and the identification of spectral scaling regimes. By associating the latter with specific interactions between vortical structures in the two fluids, an intuitive understanding of the interscale energy transfer in finite temperature, superfluid turbulence is achieved. Notably, the theoretical results are fully consistent with available experiments, allowing a deeper physical understanding of them. Finally, I discuss ways for improving the present calculations, and tackling remaining open questions.

\section{MESOSCOPIC MODEL OF FINITE TEMPERATURE SUPERFLUIDS}

I elaborate a mesoscopic viewpoint of finite temperature superfluid dynamics. This viewpoint is in direct correspondence with the emergence of superfluidity from a "condensation" type, phase transition in a simple-fluid ("normal-fluid") below a critical temperature. Here, by simple-fluid, I mean an ordinary Navier-Stokes fluid that obeys a linear stress/strain constitutive law. Indeed, above the superfluid transition temperature $T_{c}$, and for sufficiently small frequencies of mechanical excitation, ${ }^{4} \mathrm{He}$ molecules reach a local thermodynamic equilibrium and their out-of-equilibrium large scale physics are very well described by simple-fluid hydrodynamics (hence, the origin of the "normalfluid" terminology). Below the superfluid transition, however, the situation is different. As the density fraction $\chi=\rho_{s} /\left(\rho_{s}+\rho_{n}\right)$ (where $\rho_{s}$ and $\rho_{n}$ are the superfluid and normal-fluid densities) increases from zero towards unity, inhomogeneities can appear in the system in the form of linear topological defects, i.e., of quantized superfluid vortices, that form a vortex tangle. In contrast to classical vortices, the circulation of superfluid vortices is always the same, and depends only on the mass of the superfluid molecules. Progressively, as $T \rightarrow 0 \mathrm{~K}$, only the topological defects remain in the system and $\chi \rightarrow 1$. So, in a sense, the superfluid vortices behave as a large linear-particle suspension into the normal-fluid. Since superfluid vortices can grow to sizes comparable to the system-size, they remain discrete, and out-of-equilibrium, at the range of scales where the non-condensed quasiparticles form a normal-fluid continuum. Hence, the combined superfluid/normal-fluid system becomes a "complex fluid" similar to colloidal suspensions and polymeric liquids. Behind the apparent similarities with 
the dynamics of polymeric liquids, there exist crucial differences too, the most important being that polymer chains preserve their topology upon collision (become entangled) and obstruct each others motion, whilst vortices change their topology upon collision (they reconnect), and excite additional modes of motion (Kelvin waves). Nevertheless, in both cases, the main difficulties are similar: due to the presence of large-particle inhomogeneities, it is not easy to coarse grain the dynamics of the total system and obtain continuum equations for both components. Indeed, in superfluids, the well known continuum formulations of Hall-Vinen and Gorter-Mellink rely on simplified assumptions about the vortex tangle structure: the former assume it is fully structured, the latter that it is chaotic. In contrast, numerical computations show that in homogeneous, isotropic turbulence, the actual situation is in between these two important limits. ${ }^{1}$ In this work, I model the mesoscopic dynamics of superfluid vortices by developing a corresponding Langevin equation, and I couple together vortex tangle and normal-fluid flow by forming a combined Langevin-Navier-Stokes system. By solving it numerically, I obtain mesoscopic superfluid and normal-fluid velocity fields whose averages correspond to Hall-Vinen and Gorter-Mellink macroscopic formulations. Understanding the physics of the mesoscopic flow fields, provides important clues for generalizing these constitutive equations, ${ }^{12}$ and for gauging the importance of the vortex induced fluctuation stresses in the macroscopic dynamics.

\section{A. Langevin vortex dynamics}

The proposed mesoscopic Langevin dynamics for the superfluid vortex tangle read as follows:

$$
\mu_{v} \ddot{\mathbf{X}}_{v}-\mathbf{f}_{M}-\mathbf{f}_{L}-\mathbf{f}_{D}-\mathbf{f}_{F}=0 .
$$

Here, $\mathbf{X}_{v}$ is the superfluid vortex position, and $\mu_{v}$ is the superfluid mass per unit length, so that $\mu_{v} \ddot{\mathbf{X}}_{v}$ is the inertial force. There is no general consensus on the actual $\mu_{v}$ values, but according to the analysis of Baym and Chandler ${ }^{13} \mu_{v} \ll 1$.

$\mathbf{f}_{M}$ is the intervortex force per unit length as given by the standard Magnus force

$$
\mathbf{f}_{M}=-\rho_{s} \kappa \mathbf{X}_{v}^{\prime} \times\left(\mathbf{V}_{s}-\dot{\mathbf{X}}_{v}\right),
$$

where $\mathbf{X}_{v}^{\prime}$ is the unit tangent to the vortex contour and $\mathbf{V}_{s}$ is the Biot-Savart velocity field at the position on a vortex due to all other vortices including the self-interaction term,

$$
\mathbf{V}_{s}\left(\mathbf{X}_{v}\right)=\frac{\kappa}{4 \pi} \int_{\mathcal{L}} \frac{\left(\mathbf{x}-\mathbf{X}_{v}\right) \times d \mathbf{x}}{\left|\mathbf{x}-\mathbf{X}_{v}\right|^{3}} .
$$

$\kappa=h / m=9.97 \times 10^{-4} \mathrm{~cm}^{2} / \mathrm{s}$ is the quantum of circulation, with $m$ being the mass of the particles comprising the superfluid (here, ${ }^{4} \mathrm{He}$ molecules). Physically, the Magnus force is similar to the Lorentz force in the magnetostatic interaction between electrically conducting wires. Indeed, $\kappa$ acts as an analog electric current $\left(\kappa \mathrm{d} \mathbf{X}_{v}=\left(\nabla \times \mathbf{V}_{s}\right) \mathrm{dV} \leftrightarrow(\nabla \times \mathbf{B}) \mathrm{dV}=\mathbf{J d V}\right)$ and $\mathbf{V}_{s}$ as an analog magnetic field $\left(\mathbf{V}_{s} \leftrightarrow \mathbf{B}\right)$, hence, a direct analogy between Magnus and Lorentz forces $(\mathbf{J} \times \mathbf{B})$ follows. In polymer Langevin equations, the analogs of the Magnus force are the Lennard-Jones and (entropic) elastic interactions between and within chains correspondingly.

$\mathbf{f}_{D}$ is a dissipative viscous drag force, the celebrated mutual friction force of Hall and Vinen, that depends on the core structure, and couples together normal-fluid and superfluid vortices

$$
\mathbf{f}_{D}=-D_{0} \mathbf{X}_{v}^{\prime} \times\left[\mathbf{X}_{v}^{\prime} \times\left(\mathbf{V}_{n}-\dot{\mathbf{X}}_{v}\right)\right],
$$

where $D_{0}(T)$ is a temperature dependent coefficient, that has the same units as the dynamic viscosity $\left([\rho][L]^{2} /[T]\right)$. This force is analogous to the Stokes drag force in the theory of polymer and suspension dynamics. ${ }^{14}$ The linear dependence of drag on the velocity indicates a very low Reynolds number (creeping) quasiparticle flow around the vortex core, a fact consistent with the extremely small size of superfluid vortex cores, $a_{0}=1.0 \times 10^{-8} \mathrm{~cm}$. Indeed, it is well known that, at higher flow inertia, the Stokes drag law is not applicable. ${ }^{15}$

$\mathbf{f}_{L}$ is the second term that provides the coupling between Langevin and Navier-Stokes fields. It is a lift force per unit vortex length due to the Aharonov-Bohm effect for both phonon and roton parts of the normal-fluid quasiparticle spectrum, as first derived by Iordanskii (and recently rederived 
by Thompson and Stamp ${ }^{16}$ ). Due to its topological nature, the lift force does not depend on core structure, hence scales only with material properties

$$
\mathbf{f}_{L}=-\rho_{n} \kappa \mathbf{X}_{v}^{\prime} \times\left(\mathbf{V}_{n}-\dot{\mathbf{X}}_{v}\right)
$$

This force ought not to be confused with similar Magnus lift forces in classical hydrodynamics (a special instance of which is, for example, the Rubinov-Keller force ${ }^{17}$ ). The classical lift forces are a result of pressure asymmetry around the particle surface, which is induced by the rotation of a particle/vortex immersed in the flow and can be understood qualitatively by applying the Bernoulli equation. In other words, the hydrodynamic lift force is a finite Reynolds number effect. Therefore, since, as we argued above, the form of the Hall-Vinen force indicates a creeping flow around the vortex cores, one expects that a purely hydrodynamic lift force ought to be (in the range of scales of interest here) negligible in comparison with the dissipative drag. This conclusion is consistent with the microscopic (quantum field theoretical) computation of Thompson and Stamp. ${ }^{16}$ They showed that the viscous lift force tends to zero at large scales and small frequencies (Eq. (53) in their paper's supplementary material). So, it appears that two different approaches (microscopic and hydrodynamic) indicate a negligible (at the mesoscopic scales of interest here) non-Iordanskii vortex lift force. This also agrees with Sonin's conclusions in Ref. 18.

$\mathbf{f}_{F}$ is the thermal fluctuations force. Its existence can be directly inferred from the fluctuationdissipation theorem, as the counterpart of the dissipative $\mathbf{f}_{D}$ Hall-Vinen force. According to standard statistical theory, ${ }^{19}$ the components $f_{F}$ at any location on the vortex tangle are Gaussian stochastic variables with mean value zero, and time-correlator $\left\langle f_{F}\left(t_{1}\right) f_{F}\left(t_{2}\right)\right\rangle=2 D_{0}\left(k_{B} T / \ell_{F}\right) \delta\left(t_{1}-t_{2}\right)$, where $\ell_{F}$ is a length scale above which thermal fluctuation effects become important in vortex motion, and $k_{B}$ is Boltzmann's constant. The $\ell_{F}$ scale can be determined via a scaling argument: when thermal fluctuation effects become important, $k_{B} T$ should be comparable to vortex inertia. By parametrizing the latter with the vortex mass per unit length and quantum of circulation $\kappa$, it follows that $\ell_{F}=\mu_{v} \kappa^{2} /\left(k_{B} T\right)$. According to this scaling, thermal effects are more effective at larger vortex radii, since, for very small rings, the corresponding high curvatures result in very high vortex velocities that overpower thermal fluctuations. In the absence of any conclusive argument for determining the magnitude of $\mu_{v}$, and therefore gauging thermal effects correctly, I neglect the fluctuation term in the numerical calculations. Notably, Nemirovskii ${ }^{20}$ has introduced fluctuations to vortex dynamics, by working at the more microscopic Gross-Pitaevskii equation level. The origin of fluctuations in Ref. 20 is different: there, the mean field (superfluid) loses energy, which is spent in exciting the quantum fluctuations of the vacuum state into particles that comprise the normalfluid. ${ }^{19}$ This is the main dissipation process in the superfluid, and together with the accompanying fluctuations in the number of the excited particles lead to a dissipative, stochastic Gross-Pitaevskii equation. For comparison, in the mesoscopic dynamics proposed here, the fluctuations originate in the scattering of existing normal-fluid quasiparticles by the vortex cores.

Before leaving the vortex dynamical part of the model, it must be remarked that some forces are possibly missing, for example, forces due to the inelastic scattering of quasiparticles by a vortex because of Kelvin waves excitation (I am grateful to Joe Vinen for indicating this complication), or entropic forces due to thermal fluctuations at subgrid scales, when computational complexity constrains the discretization length along the vortices to be much larger than $\ell_{F}$ (an example of such a force is entropic elasticity in polymers). However, the excellent agreement between the predictions of the current model and Particle Image Velocimetry data ${ }^{21,22}$ suggests that the basic physics are adequately captured. Definitely, there could be special experiments, where the fluctuating aspects of the dynamics or even vortex inertia could be manifested and in need of modeling, but this does not appear (at present) to be the case in available turbulence experiments. Summarizing the vortex tangle dynamics, one notes the (perhaps surprising) similarities between superfluids and soft matter physics. The unification of these topics via Langevin dynamics, that I attempt here, could indicate ways of improving the modeling of superfluid flows via cross-fertilization of these traditionally separate disciplines. Notably, the key physical quantities of the outlined viewpoint are the normalfluid velocity and the superfluid vortex position. The mathematical model primarily incorporates an intuition about vortex motion, and the superfluid velocity is a secondary, derived quantity. 


\section{B. Vortex reconnections}

A crucial limitation of the above Langevin equation is its inability to resolve vortex reconnections. Indeed, reconnections take place at microscopic (of the order of the vortex core) scales which are absent in the mesoscopic treatment. This situation is typical in mesoscopic studies of complex fluids, where (for example) polymer entanglements ${ }^{23}$ and colloid collisions need to be modeled phenomenologically at the Langevin equation level. In superfluids, the first such phenomenological model of vortex reconnection was proposed by Schwarz. ${ }^{8}$ My method is a close variant of Schwarz's ${ }^{8}$ approach. In particular, let $\left\{\mathbf{X}_{v}^{i-1}, \mathbf{X}_{v}^{i}, \mathbf{X}_{v}^{i+1}\right\}$ and $\left\{\mathbf{X}_{v}^{j-1}, \mathbf{X}_{v}^{j}, \mathbf{X}_{v}^{j+1}\right\}$ be any two sequences of discrete vortex points $(i-1 \neq i \neq i+1 \neq j-1 \neq j \neq j+1)$. The increasing $i$ or $j$ indices follow the vorticity direction along the contours. In numerical calculations, the discrete vortex points are separated by the effective cut-off scale $\delta_{v}$ of $\mathbf{X}_{v}$-fluctuations which is of the order of the grid size along the vortices $\Delta \xi, \Delta \xi / \alpha<\left|\mathbf{X}_{v}^{i}-\mathbf{X}_{v}^{i-1}\right|<\alpha \Delta \xi$. Here, $\xi$ is the arclength parametrization along the vortices, and $\alpha>1$ is a computational parameter allowing a small variability in the discretization length. In the computations, $\alpha=1.65$. Next, define the intervortex spacing scale $\delta_{i v}=\sqrt{\mathcal{V}_{s} / L}=\Lambda^{-1 / 2}$ (with $\mathcal{V}_{s}$ the system volume, $L$ the vortex tangle length, and $\Lambda=L / \mathcal{V}_{s}$ the vortex line density). Hence, the reconnection algorithm and the accompanying topological change becomes

$$
\begin{aligned}
& \left\{\mathbf{X}_{v}^{i-1}, \mathbf{X}_{v}^{i}, \mathbf{X}_{v}^{i+1}\right\} \wedge\left\{\mathbf{X}_{v}^{j-1}, \mathbf{X}_{v}^{j}, \mathbf{X}_{v}^{j+1}\right\} \wedge\left[\left|\mathbf{X}_{v}^{i}-\mathbf{X}_{v}^{j}\right|<\beta \min \left(\Delta \xi, \delta_{i v}\right)\right] \longrightarrow \\
& \left\{\mathbf{X}_{v}^{i-1}, \mathbf{X}_{v}^{i}, \mathbf{X}_{v}^{j+1}\right\} \wedge\left\{\mathbf{X}_{v}^{j-1}, \mathbf{X}_{v}^{j}, \mathbf{X}_{v}^{i+1}\right\} .
\end{aligned}
$$

The difference between Schwarz's ${ }^{8}$ method and the present one is the inclusion of the $\delta_{i v}$ scale in the reconnection criterion. This is deemed necessary when very dense tangles are produced in a computation (as is always the case in turbulence), in order to avoid the proliferation of spurious reconnections. $\beta$ is an important, smaller than unity, computational parameter $(\beta=0.3$ in the present calculations). It controls two crucial physical effects: (a) the rate with which reconnections are occurring, that is, the smaller the $\beta$, the smaller the reconnection rate, and (b) the rate with which reconnections remove vortex length from the tangle. The latter is a desirable feature, since (during reconnections) vortex kinetic energy is transformed into acoustic energy which (via the mixed fluctuation/mean-field terms in the governing equation for the mean quantum field value) create particles in the normal-fluid. Certainly, the physics of such processes are not modeled in the incompressible vortex dynamics of the present Langevin equation, thus removing vortex length during reconnections is a phenomenological, albeit crude, way of mimicking the above microscopic quantum mechanical process. In addition, another source of vortex length loss in the calculations is the removal of rings with three or less vortex points from the system. That the combination of the above reconnection model with the particular $\alpha$ and $\beta$ values work as expected in practice was shown in Ref. 24. Indeed, in this work, in order to keep a constant tangle length during a $T=$ $0 \mathrm{~K}$ computation, thus, to compensate for reconnection induced losses, an energy injection into the system in the form of superfluid vortex rings had to be applied. A systematic investigation of these and similar issues is available in Ref. 25.

\section{Navier-Stokes fluid dynamics}

In order to complete the physical model, the Navier-Stokes dynamics for the normal-fluid need to be introduced. These are quite standard, apart from the added forces that couple the normal-fluid with the superfluid vortices, and are the exact opposites of the $\mathbf{f}_{L}, \mathbf{f}_{D}$ forces acting on the latter

$$
\begin{gathered}
\nabla \cdot \mathbf{V}_{n}=0 \\
\frac{\partial \mathbf{V}_{n}(\mathbf{x}, t)}{\partial t}+\nabla\left(\frac{p}{\rho_{n}+\rho_{s}}+\frac{\mathbf{V}_{n} \cdot \mathbf{V}_{n}}{2}\right)-\mathbf{V}_{n} \times\left(\nabla \times \mathbf{V}_{n}\right)-\frac{\mu}{\rho_{n}} \nabla^{2} \mathbf{V}_{n}- \\
\kappa \int_{\mathcal{L}} d \xi\left[\mathbf{X}_{v}^{\prime} \times\left(\mathbf{V}_{n}-\dot{\mathbf{X}}_{v}\right)\right] \delta\left(\mathbf{x}-\mathbf{X}_{v}\right)- \\
\frac{D_{0}}{\rho_{n}} \int_{\mathcal{L}} d \xi\left\{\mathbf{X}_{v}^{\prime} \times\left[\mathbf{X}_{v}^{\prime} \times\left(\mathbf{V}_{n}-\dot{\mathbf{X}}_{v}\right)\right]\right\} \delta\left(\mathbf{x}-\mathbf{X}_{v}\right)-\left(\epsilon / 3 V_{n}^{2}\right) \mathbf{V}_{n}=0 .
\end{gathered}
$$


Here, $p(\mathbf{x}, t)$ signifies the scalar pressure field that enforces incompressibility, $\epsilon=-v\left\langle\mathbf{V}_{n} \cdot \nabla^{2} \mathbf{V}_{n}\right\rangle$ is the rate of kinetic energy dissipation, $V_{n}^{2}=\frac{1}{3}\left\langle\mathbf{V}_{n} \cdot \mathbf{V}_{n}\right\rangle$ is the mean square of turbulence fluctuations, and $v=\mu / \rho_{n}$ is the kinematic viscosity of the normal-fluid. The delta functions are employed to indicate that the coupling between normal-fluid and vortex tangle takes place exclusively along vortex contours. It is important to note here that the fourth, fifth, and sixth terms have in front a coefficient with units of kinematic viscosity. Due to the dissipative nature of the sixth (Hall-Vinen) force, it is appropriate to call $D_{0} / \rho_{n} \equiv \lambda$ the "mutual-friction viscosity" acknowledging that it could simply be a "renormalized" version of the standard kinematic viscosity $v$. Equally important, since the fifth term is not dissipative in nature, it scales with the quantum of circulation that is connected to inertial aspects of the superfluid vortices. Indeed, $\kappa$ is a constant "vortex current" that allows the vortex to sense/interact with the normal-fluid flow. Consequently, the actual value of $\kappa$ gauges the coupling between vortices and flow in the same fashion that gauges the coupling of vortices with one another (and themselves) in the Magnus term of the Langevin equation. Therefore, in addition to the standard Reynolds number $R e=U^{*} D^{*} / v$ that gauges inertia against viscous forces, one can define two more nondimensional numbers, $U^{*} D^{*} / \kappa$ that compares normal-fluid inertia with the Iordanskii force, and $U^{*} D^{* / \lambda}$ that gauges normal-fluid inertia against the mutual-friction force. As a result, by performing a harmless redefinition of the pressure $p /\left(\rho_{n}+\rho_{s}\right) \rightarrow p$, one can scale the normal-fluid equation with three nondimensional numbers. The last term of the equation (also known as Lundgren force ${ }^{26-28}$ is a force that mimics the injection of energy in a normal-fluid turbulent flow. In real-life situations, there are many ways to stir up a fluid, for example, by having it interact with a solid or a gravitational/electromagnetic field. The Lundgren force is a method for achieving a controllable injection of energy in a turbulent flow without unnecessary real-life complications. It has been shown $^{1,27,28}$ that the Lundgren force is consistent with Kolmogorov's inertial range energy spectrum scaling so it does not "contaminate" the statistical structure of normal-fluid turbulence. This force is introduced in the Navier-Stokes equation for the following reason: the structure of pure normalfluid turbulence is identical to that of turbulence in a simple-fluid, which is a topic of numerous investigations and many of its aspects are very well understood. In superfluids, it is the physics of the coupling between normal-fluid and superfluid vortices that is the great unknown, in particular in the context of their effects on both normal-fluid turbulence and superfluid vortex tangle structure. So, there is a need to isolate the effects of the mutual-friction and lift coupling forces on the energetics of normal-fluid turbulence, and this is the sole purpose of the Lundgren force here. Its function is to inject, at each time instant, the exact amount of kinetic energy that is dissipated by the $\nu \nabla^{2} \mathbf{V}_{n}$ term. Thus, since the other (non-coupling related) forces in the Navier-Stokes equation conserve energy, any normal-fluid energy dynamics in the computations are due entirely to its coupling with the vortices, and the energy transfer between the two subsystems because of their coupling can be explicitly demonstrated. Moreover, since the Lundgren force does not alter spectral scalings, ${ }^{1,27,28}$ any normal-fluid turbulence deviation from standard simple-fluid turbulence physics can be safely attributed to the effects of the mutual-friction and lift force couplings.

\section{NUMERICAL RESOLUTION, METHODS, AND CODES}

I discuss the design of finite temperature superfluid computations from the points of view of spatial and temporal resolution, fluid and vortex numerical methods, as well as key algorithmic difficulties.

\section{A. Spatial and temporal resolution}

Setting up well resolved, finite temperature, superfluid turbulence calculations is not a trivial task. There are a number of new scales not present in simple-fluid turbulence, and one needs to consider carefully the resolution of both normal-fluid velocity and superfluid vortex fluctuations, in order to capture all important physics. There are three important scales in the system: the cut-off scale $\delta_{f}$ of normal-fluid velocity fluctuations, the cut-off scale $\delta_{v}$ of vortex contour fluctuations, and, in numerical calculations, the regularization scale $\delta_{\delta}$ of the mutual friction and lift-force delta-functions 
in the Navier-Stokes equation. Since the smoothing of the delta-function singularities introduces a de facto effective cut-off scale $\delta_{\delta}$ in the dynamics, the resolution of all important physics is possible if, and only if, $\delta_{\delta}<\min \left(\delta_{f}, \delta_{v}\right)$. The key question then is what is $\delta_{f}$ and $\delta_{v}$ ?

The combination of Langevin dynamics and reconnection model allows the description of a key dynamical vortex tangle process, the Kelvin wave cascade. In particular, the collision of superfluid vortices and their subsequent reconnections induces contour waves along the filaments (Kelvin waves) which cascade kinetic energy from the reconnection scale (of the order of the curvature of the reconnecting vortices) to smaller scales. ${ }^{29-33}$ In an inviscid, incompressible, pure superfluid, the Kelvin waves cascade would extend all the way to the vortex core, but actual superfluid vortices are compressible, so, as they fluctuate, they radiate sound and lose their kinetic energy. ${ }^{34}$ At very low temperatures, sound radiation would be the dominant damping process, but in the temperatures of interest here, there is also dissipative mutual friction action which leads to higher $\delta_{v}$ values than those expected on the grounds of sound emission alone. The calculation of Ref. 35 is instrumental in this respect: there, an initially stationary normal-fluid at $T=1.3 \mathrm{~K}$ self-consistently interacts with a tangle of (initially) randomly oriented superfluid vortices. The reconnections that occur in the tangle generate strongly damped Kelvin waves, and a fractal tangle geometry. Since the normal-fluid is forced by mutual-friction along a complicated, fractal contour, it develops fluctuations itself. The latter are characterized by very small Reynolds number, $R e \approx 10^{-3}$, and their energy spectrum scales like $k^{-2.2}$ terminating at a cut-off scale of the same order as the superfluid intervortex distance $\delta_{i v}{ }^{35}$ Indeed, in Ref. 35, the intervortex distance wavenumber is $k_{\delta_{i v}} \approx 277 \mathrm{~cm}^{-1}$, and coincides with the end of the normal-fluid spectrum. It is important to note here, that this is a genuine cut-off since the smallest wavenumber resolved in the calculation is $k=641 \mathrm{~cm}^{-1}$. This implies that the Kelvin waves cascade is damped at about $\delta_{v}=\delta_{i v}$, a conclusion supported further by the very smooth vortex contours (Fig. 3 in Ref. 35). Indeed, the cascade does not extend well beyond $\delta_{i v}$, because, in this case, the Kelvin waves would have excited normal-fluid fluctuations there, instead of the observed cut-off. Noting that the Kelvin waves cascade is expected to start at the curvature scale typical of reconnections, and taking into account that in dense tangles, as that in Ref. 35, the reconnecting scale is of the order of $\delta_{i v}$, one concludes that the Kelvin waves cascade is damped by mutual friction at about the scale of its generation. A second, equally important conclusion drawn in Ref. 35 is that, in contrast to turbulence in simple-fluids, the sub-turbulence normal-fluid velocity scales are not smooth, but are characterized by small Reynolds number fluctuations that terminate at a cut-off scale of the same order as the superfluid intervortex distance (which can be much smaller than the smallest scale of turbulent/inertial fluctuations in the normal-fluid).

The above allow a self-consistent, physically sound design of finite temperature superfluid turbulence computations as follows: choose a numerical discretization length $\Delta x$ for the NavierStokes equation taking into account computational complexity limitations. Then, for consistency, choose $\delta_{\delta}=\Delta x\left(\delta_{f} \geq \Delta x\right)$. When deciding the Taylor Reynolds number for normal-fluid turbulence, ensure that the Kolmogorov scale $\eta$ in the initial turbulent velocity field is $\eta>\Delta x$. In this way, not only the range of scales of turbulence/vortex-tangle coexistence is well resolved, but there is also available a computational scale-range for resolving the sub-turbulence low Reynolds number normal-fluid fluctuations of Ref. 35. Capturing the latter regime is a good indication that a superfluid turbulence calculation is well resolved. Next, choose the superfluid discretization length along the filaments $\Delta \xi$ (where $\xi$ is the arclength parametrization along the vortices) to be $\Delta \xi=\Delta x=$ $\delta_{\delta}\left(\delta_{v} \geq \Delta \xi\right)$. This is because there is no reason to resolve the vortex contours (and associated Kelvin waves) at scales smaller than the $\delta_{\delta}$ regularization scale, since there are no normal-fluid dynamics at those scales for the Kelvin waves to interact with. Certainly, since the sub-turbulence low Reynolds number, normal-fluid fluctuations terminate at the intervortex scale $\delta_{i v}$, i.e., $\delta_{f} \approx \delta_{i v},{ }^{35}$ and $\delta_{v} \approx \delta_{i v},{ }^{35}$ one ought to suspend the computation when $\delta_{i v} \approx \delta_{\delta}$ so that the calculations do not become under-resolved. In summary, when setting turbulence calculations in this way, one captures all relevant dynamical regimes and only the attainable superfluid vortex tangle densities are limited. Indeed, both turbulent and low Reynolds number fluctuations in the normal-fluid are resolved, and the superfluid vortex tangle is allowed to form any large scale organization/pattern, and develop Kelvin waves cascade at smaller scales. In this respect, the self-consistency of physics and numerics also needs to be stressed, since as discussed in Ref. 36, the numerical vortex dynamical method 
introduces a cut-off of superfluid velocity at the scale $\Delta \xi$, which is harmless if, and only if, it is assured that $\Delta \xi \leq \delta_{v}$, which is what this setup achieves.

Regarding time resolution of normal-fluid flow, it is not adequate to resolve only the turbulent normal-fluid fluctuations. One also needs to resolve the low Reynolds number fluctuations of Ref. 35, and this demands a viscous time step, i.e., $\Delta t \leq \min \left(\min \left[\gamma \Delta x / V_{n}(\boldsymbol{x})\right], \Delta x^{2} / 6 v\right)$, where $\gamma$ is the CFL number. The superfluid physics also impose restrictions on the time step, by the need to resolve the fastest Kelvin waves on the vortices. Since the smaller a Kelvin's wave wavelength, the faster it propagates, only the smallest Kelvin waves need to be considered. These have wavelength $\lambda_{K}=2 \Delta \xi$, and their group velocity follows from the general formula $V_{\lambda_{K}}=\left(\kappa / 2 \lambda_{K}\right) \log \left(\lambda_{K} / 2 \pi a_{0}\right)$. Then $\Delta t \leq \lambda_{K} / V_{\lambda_{K}}$. In the calculations presented here, the normal-fluid time step is the more restrictive one.

\section{B. Numerical methods and algorithms}

I employ a standard, projection method for the Navier-Stokes equation. ${ }^{36}$ The non-standard element is the smoothing of the delta function singularities in the coupling terms, and this is achieved with the use of Heaviside functions. ${ }^{1}$ For the vortices, I renormalize the self-interaction velocity divergence in the Biot-Savart law by employing the velocity of a ring with radius the local radius of curvature, and apply the method of Winckelmans and Leonard for evaluating velocity contributions because of all other points. ${ }^{37}$ For the latter, an effective vortex core radius equal to $\Delta \xi$ is employed. ${ }^{37}$ As remarked earlier, in the numerical analysis of vortex dynamics, thermal fluctuation effects are neglected. In addition, high frequency vortex oscillations are not of interest, and are not resolved. In other words, the vortex dynamics are solved in the (so called) diffusive limit, that is, with numerical time steps $\Delta t \gg \tau_{R}$, where $\tau_{R}=\mu_{v} / D_{0}$ is the inertial relaxation time scale. In this way, without compromising physical accuracy, one overcomes the problem with the uncertainty in the $\mu_{v}$ values, since, in the diffusive limit, vortex inertia can be neglected, and one can use the vortex dynamical scheme introduced in Ref. 38 and further modified in Ref. 1. Finally, in contrast to the Navier-Stokes part, the algorithms of the vortex dynamical part are difficult exercises in computational geometry. In writing code for reconnecting dynamics, emphasis must be placed from the outset on having the correct data structures. Detecting and performing reconnections is a demanding algorithmic task, because it is accompanied by changes in the tangle topology and the number of vortices in the system. It is best if extensive tests of such algorithms are performed before undertaking any actual physics computations.

\section{SETTING UP A FINITE TEMPERATURE SUPERFLUID TURBULENCE}

The working fluid is superfluid ${ }^{4} \mathrm{He}$ at $T=1.3 \mathrm{~K}$. The values of the various experimental material properties/parameters are available in Ref. 1. The computational domain is a periodic cube of size $l_{b}=0.1 \mathrm{~cm}$, thus, the size of the largest resolved eddies is $l_{e} \approx 0.5 \times l_{b}$. Within this domain, we set up a steady state, homogeneous, isotropic normal-fluid turbulence with Reynolds number $R e=V_{n} l_{e} / \nu=612$ (the turbulence intensity $V_{n}=28.52 \mathrm{~cm} \mathrm{~s}^{-1}$ ). This steady state is established in a separate, pure normal-fluid computation, where an initially random normal-fluid velocity field with Gaussian statistics ${ }^{39}$ is allowed to evolve under Navier-Stokes dynamics whilst the Lundgren force injects kinetic energy at a specified, constant rate. ${ }^{27,28}$ The level of energy injection is determined by the value of the targeted steady state Taylor Reynolds number. The latter, in turn, is dictated by combined computational complexity considerations, since, in a superfluid computation, the demanding resolution of fully developed normal-fluid turbulence is only partially responsible for the computational load, and one needs to correctly anticipate the size of the superfluid vortex problem too, in order to design manageable computations. Employing the relation $\lambda / l_{e}=\sqrt{15} R e^{-1 / 2}$, where $\lambda$ is the Taylor microscale, one finds $R e_{\lambda}=95$. I employ an $128^{3}$ computational mesh, i.e., the grid size is $\Delta x=0.781 \times 10^{-3} \mathrm{~cm}$ which corresponds to wavenumber $k_{\Delta x}=1280$. The scaling relation $\eta$ $\approx l_{e} / R e^{3 / 4}$ suggests $\eta \approx 0.4063 \times 10^{-3} \mathrm{~cm}$ for the Kolmogorov scale. Comparing $\eta$ with $\delta x$ indicates a well resolved turbulence computation, a conclusion supported by the computational results. As discussed in Sec. III, I choose $\delta_{\delta}=\Delta x$, and $\Delta \xi=2 \Delta x$. Moreover, since the smallest intervortex 
spacing scale $\delta_{i v}$ recorded in the computations is $\delta_{i v}=2.18 \times 10^{-3} \mathrm{~cm}>\Delta x$, the numerical setup is adequate for the resulting flow. This is also verified by the computational results, which indicate that the $k^{-2.2}$ scaling regime of Ref. 35 is adequately resolved. The initial state of the superfluid vortex tangle consists of 40 rings randomly oriented and positioned within the box. Their radii are randomly distributed between $r_{\max }=0.25 l_{b}$ and $r_{\min }=0.75 r_{\max }=0.1875 l_{b}$. Notably, as verified in numerous computations of superfluid turbulence, the results do not depend on the initial conditions in the superfluid, since the tenths of thousands of reconnections that occur during the computation (in combination with the random character of the turbulent normal-fluid velocity) create fast a homogeneous, superfluid vortex tangle. Hence, the initial conditions provide only a reasonable vortex configuration in order to start the computation. Notably, the large-eddy turnover time in the normal-fluid is of the order of $\tau_{e}=l_{e} / V n=0.175 \times 10^{-3} \mathrm{~s}$, thus, taking into account that the total computation time is $t_{f}=0.0619 \mathrm{~s}$, we have $t_{f} \approx 35 \tau_{e}$. The typical time step in the computations is of the order $\Delta t \approx 10^{-6}-10^{-5} \mathrm{~s}$.

\section{DECAYING FINITE TEMPERATURE SUPERFLUID TURBULENCE}

The results reveal the spectral structure of turbulence in the two fluids. Some of the resulting scaling laws do not have the desirable, large extension in wavenumber range because, due to computational complexity, it is not realistic, at present, to compute very large systems. Nevertheless, by putting together physical arguments, and by combining the present results with other previously obtained ones, I provide here an account of normal-fluid and superfluid energy scalings in finite temperature quantum fluids. This account is going to be systematically examined in future, more potent, massively parallel computations. Notably, the introduction of the Lundgren force, does not allow the flow to decay under the combined action of viscous dissipation and acoustic radiation mechanisms. Because of this, I do not draw any conclusions in this work about the temporal structure of turbulence. All conclusions involve spectral analysis of the spatial structure, which is computed from first principles, and is not deformed by the Lundgren force. The resulting conclusions are applicable to the spatial structure of all homogeneous, isotropic, superfluid turbulence flows. Thus, it applies to counterflow turbulence only when the mean flow does not cause drastic departures from isotropy in the vortex tangle, and is relevant to vibrating-object generated turbulence, insofar as the latter occurs at finite temperatures.

\section{A. Evolution of global quantities}

As shown in Fig. 1 (left), the initial condition for the normal-fluid presents a low $k$ Kolmogorov $k^{-5 / 3}$ regime. The initial superfluid velocity is negligible in comparison with the normal-fluid velocity,
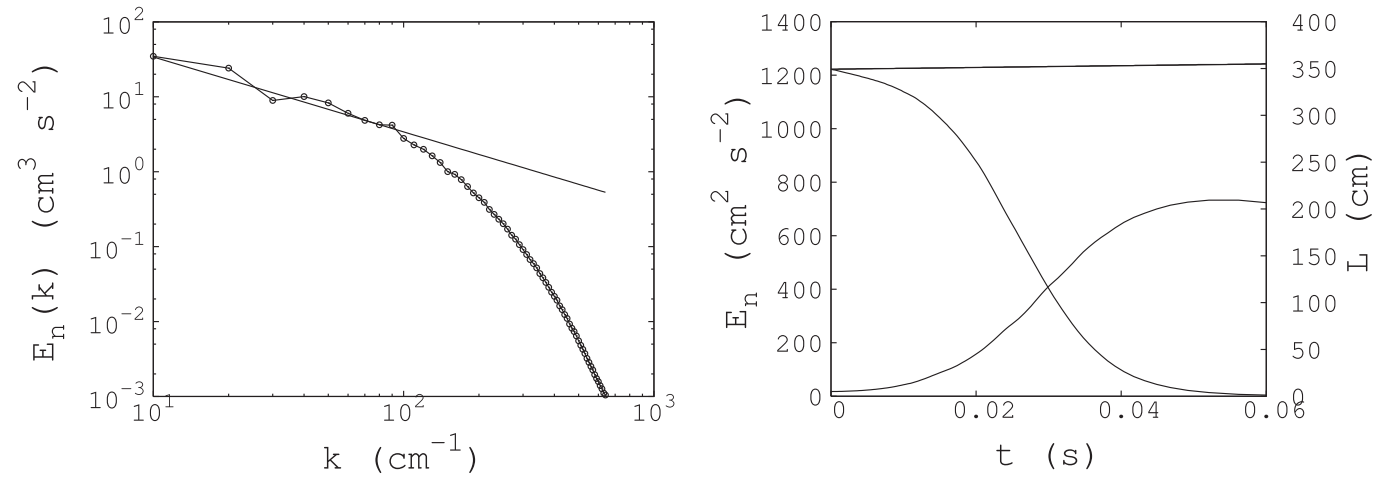

FIG. 1. Left: Initial energy spectrum $E_{n}(k)$ of the homogeneous isotropic turbulence in the normal-fluid. A Kolmogorov $k^{-5 / 3}$ scaling regime (straight line) is observed over a decade in wavenumber space. Evidently, the dissipation regime of the spectrum is also well resolved. Right: Decaying normal-fluid kinetic energy $E_{n}$ and growing superfluid vortex tangle length $L$ versus time. The straight horizontal line shows $E_{n}$ in an accompanying computation, where the superfluid is not allowed to react back on the normal-fluid (and viscous action is counterbalanced by the Lundgren force). 
and this is also the case for the superfluid kinetic energy, despite the density ratio $\rho_{s} / \rho_{n}=21.257$, for $T$ $=1.3 \mathrm{~K}$. Indeed, defining a normal-fluid eddy circulation $\Gamma_{\ell} / v=R e_{\ell}$, where $\Gamma_{\ell}$ is the circulation of an eddy of size $\ell$ and $R e_{\ell}$ is the corresponding Reynolds number, it follows $\Gamma_{\ell_{e}}=1430 \kappa\left(R e_{\ell_{c}}=612\right)$, and $\Gamma_{\ell_{\eta}}=2.33 \kappa\left(R e_{\ell_{\eta}}=1\right) . \kappa=9.97 \times 10^{-4} \mathrm{~cm}^{2} / \mathrm{s}$ is the quantum of circulation. Evidently, the large normal-fluid eddies are much more energetic than the superfluid ones. At $t=0$, the two fluids start interacting via mutual-friction and lift forces. After an initial transient (Fig. 1, right), the normal-fluid energy decays linearly in time, until, following a second transient, it relaxes (close to the final time $t_{f}=0.0619 \mathrm{~s}$ ) to an asymptotic low Reynolds number state. This normal-fluid energy decay is a purely mutual-friction, lift-force effect, because, according to the logic of the setup, the viscous losses are completely counterbalanced by the action of the Lundgren force. In order to demonstrate this point, I also show in Fig. 1 (right), the results of a normal-fluid calculation without any coupling to the superfluid. Evidently, the Lundgren force acts as expected, keeping the normal-fluid energy approximately constant. It is important to compare the strength of the effects of coupling forces with the strength of the effects of viscous forces. For this purpose, I have also allowed the $t=0$, pure normal-fluid turbulence state to decay from its initial condition because of viscous action only. According to the results, the coupling forces need nine times more time than the viscous forces, in order to remove $90 \%$ of the initial kinetic energy. Intuitively, this is understood by noting that mutual-friction is proportional to normal-fluid velocity values, but viscous force is proportional to their second derivatives (which are expected to be much larger, since turbulence fluctuations are characterized by large velocity gradients). These indicate a weak coupling between the two fluids in comparison with the other terms in the Navier-Stokes equation. Indeed, the coupling forces are weaker than the viscous forces, which, in turn, are much weaker than the inertial and pressure gradient terms for $R e_{\ell_{e}}=612$. The superfluid has similar with the normal-fluid physics, i.e., inertial forces whose strength scales with the quantum of circulation, and the mutual-friction and lift force couplings that pump energy into it. The important difference is that, instead of the viscous dissipation that transforms kinetic energy into heat in the normal-fluid, there is sound emission from vortex vibrations that transforms kinetic energy into acoustic energy in the superfluid. This energy sink is not included in the analytical vortex dynamics, and is modeled at the computational level via length loss due to reconnections, and small vortex ring removal. Therefore, at every time-instant, the normal-fluid loses energy to the superfluid because of their coupling, whilst the superfluid gains the energy transfered from the normal-fluid, whilst losing energy due to reconnections-induced vortex length reduction.

A key observation is that the two fluids reach a final state with very low energy levels in the normal-fluid. The initial normal-fluid kinetic energy is spent in order to eventually create a dense chaotic vortex tangle that coexists with a low Reynolds number normal-fluid flow, similar to the vortex tangle and flow of Ref. 35. Notably, as discussed at great length below, this effect does not exclude a transient, i.e., at an earlier time, organization of the vortex tangle at low $k$, where the inertial range of the normal-fluid resides. Such an organized vortex tangle at low $k$ that comes into kinetic equilibrium with the normal-fluid at the same scales cannot be a stationary solution of the dynamics. This is because vortex reconnections spent superfluid kinetic energy, and since this loss is not compensated, it drives superfluid energy towards smaller values. This leads to further transfer of energy from the normal-fluid to the superfluid, until the $k$-extension of the inertial range in the normal-fluid is diminished, and with it also the organization of the vortex tangle, leaving behind the final chaotic tangle state of Ref. 35. At even later times, the continuous (reconnection related) sink of total energy will eventually drive the whole system into a quiescent state with no vortices, zero normal-fluid flow, and higher temperature. Notably, in a similar fashion with the incompressible dynamics of simple-fluids, the heating of the quantum fluid is not accounted for in the incompressible dynamics that we solve here.

\section{B. Spectral analysis of normal-fluid turbulence}

The normal-fluid spectra are shown in Fig. 2 (left). In order to indicate the progressive reduction of the $k$-extension of the Kolmogorov regime with time, I have superimposed the spectra at various times, matching the low $k$ energy levels by multiplying them with appropriate factors (reported in 

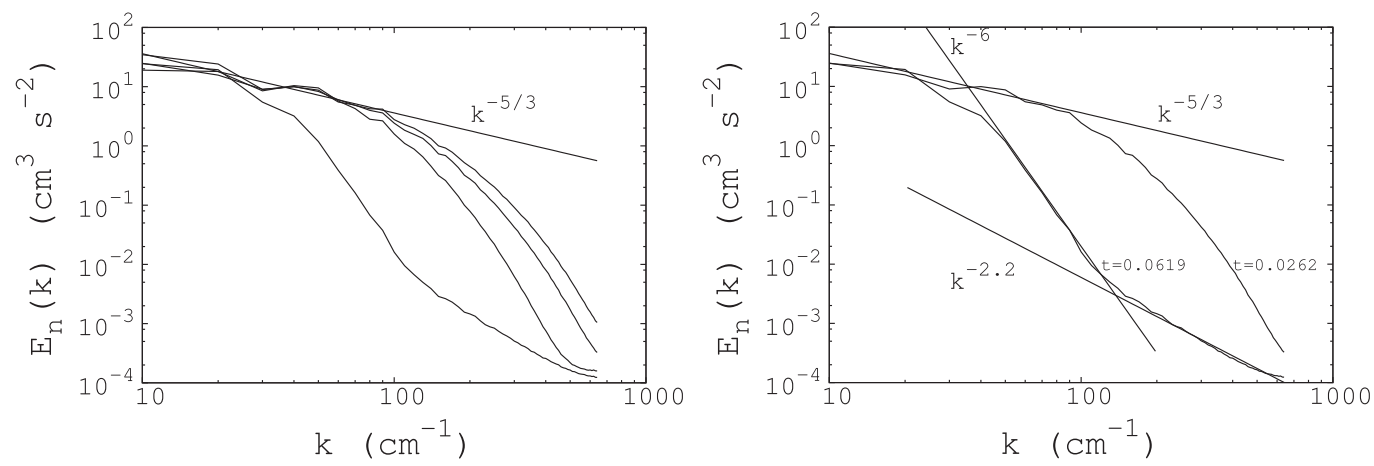

FIG. 2. Left: Superimposed normal-fluid energy spectra at times $t=0, t=0.0262 \mathrm{~s}, t=0.0363 \mathrm{~s}$, and $t=0.0619 \mathrm{~s}$ (right to left). In order to match the low wavenumber energy levels, I multiplied later time spectra by the factors $1.7,5$, and 180 . The straight line indicates the slope of Kolmogorov's $k^{-5 / 3}$ scaling. As the normal-fluid loses its kinetic energy via its coupling to the superfluid, its inertial range extension in $k$-space shrinks towards low $k$. The corresponding $\left(t-k_{\delta_{i v}}\right)$ pairs are: $(0,71)$, $(0.0262,293),(0.0363,405),(0.0619,453)$. Right: Normal-fluid spectra at $t=0.0262 \mathrm{~s}$ and $t=0.0619 \mathrm{~s}$. In contrast to the $t=0.0262 \mathrm{~s}$ case, the $t=0.0619 \mathrm{~s}$ spectrum does not present a substantial inertial range. There is an intermediate, steep, $k^{-6}$ viscous scaling regime, that bridges the more energetic large scale eddies with a low Reynolds number fluctuating flow exhibiting a $k^{-2.2}$ energy scaling. ${ }^{35}$

caption). At $t=0$, a low $k$ Kolmogorov regime over a decade is observed. Since the mutual-friction force is proportional to the normal-fluid velocity, its physics can be expected to be similar, in many respects, to the physics of the Lundgren force. The Lundgren force pumps energy in the normalfluid whilst preserving its energy spectral scaling, and, according to the results, mutual-friction is removing energy whilst also preserving the energy scaling. Indeed, at $t=0.0262 \mathrm{~s}$, i.e., well into the linear (normal-fluid) energy decay period, the Kolmogorov scaling regime remains intact (modulo an overall decrease in energy levels). Moreover, since mutual-friction effects are much weaker than inertial effects (because mutual-friction is weaker than the viscous force and the Reynolds number is high), the physics of the inertial range in the normal-fluid are not expected to differ greatly from the physics of simple-fluid turbulence. Hence, the persistence of strong normal-fluid nonlinearity at low $k$ implies vortex stretching, and the existence of small scale, high enstrophy normal-fluid vortex tubes that generate superfluid vortex length dynamos, ${ }^{37}$ whilst getting damped in the process. This effect is demonstrated in Fig. 3. As shown in Ref. 37, these dynamos include the generation of spirallike superfluid vortices around the small scale normal-fluid tubes, and of aligned, superfluid vortex bundles within them. The latter effect is much weaker than the former. The spiral vortices originate in vortex instabilities that grow, due to the coupling forces, to large radii that are comparable to the size of the large eddies in the normal-fluid. This large scale growth of superfluid vorticity is the physical mechanism responsible for mutual-friction induced energy removal from the normal-fluid.

The self-similar decay continues until the Reynolds number in the normal-fluid cannot support a Kolmogorov regime any further. Indeed, at $t=0.0363 \mathrm{~s}$, the inertial range's extension in $k$ space starts diminishing fast, and the flow enters a transient towards a low Reynolds number flow. When, at $t=0.0619 \mathrm{~s}$, this transient is over, there is no Kolmogorov regime in the normal-fluid spectrum. Instead, both fluids find themselves in a low kinetic energy state. This state is none other than the flow of Ref. 35, since, as discussed in Sec. III A, in every normal-fluid turbulent flow there are sub-turbulence fluctuations with a $k^{-2.2}$ energy scaling. These fluctuations comprise a low-Reynolds number flow generated in a normal-fluid by a more energetic superfluid, and are characterized by the triple vortex structures of Ref. 40. Indeed, since turbulent normal-fluid fluctuations have a steep high $k$ cut-off, one can always find a large enough $k$ where the superfluid (because of its vortical topological defects) is more energetic than the normal-fluid. It should be stressed, that the non-trivial low Reynolds number normal-fluid flow structure is not an inertial effect, and its apparent complexity is solely due to the forcing of the normal-fluid along a fractal vortex tangle contour. ${ }^{35,41}$ As the normal-fluid turbulence cut-off shifts to lower $k$ in Fig. 2 (left), the creeping flow regime becomes more noticeable, and its $k^{-2.2}$ scaling is evident at $t=0.0619 \mathrm{~s}$, Fig. 2 (right). At the same time, an 

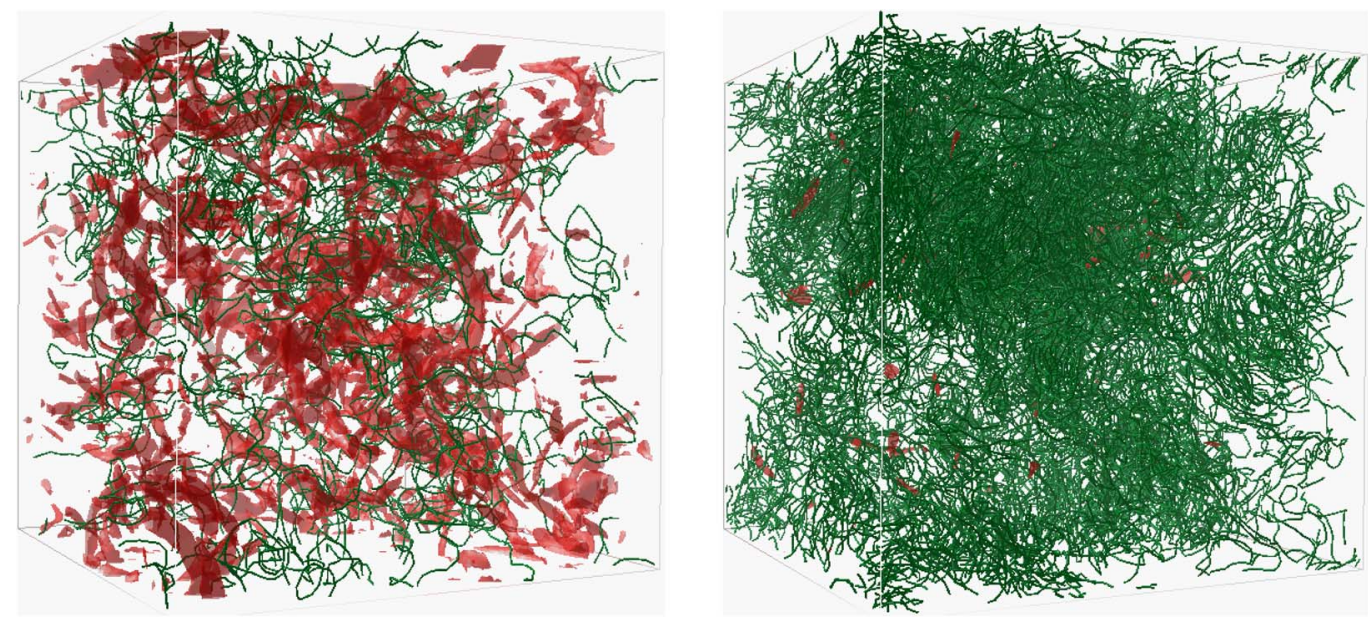

FIG. 3. Isosurfaces of intense normal-fluid vorticity (red tubes) and superfluid vortices (green lines) at $t=0.0113 \mathrm{~s}$ (left) and $t=0.0262 \mathrm{~s}$ (right). The earlier time results refer to the initial transient stage before the period of linear normal-fluid energy decay. The subsequent growth of superfluid vorticity damps the intense, small scale vorticity structures in the normal-fluid.

intermediate viscous spectrum regime with a steep $k^{-6}$ scaling appears in between the now-defunct inertial and creeping flow regimes.

\section{Spectral analysis of superfluid turbulence}

As discussed in Sec. III A, the Kelvin waves cascade appears to be damped close to the intervortex scale $\delta_{i v}$. This conclusion is indicated by the results in Ref. 35, where the normal-fluid sub-turbulence fluctuations terminate at $\delta_{i v}$ indicating no significant superfluid structure beyond this scale, a conclusion also supported by the accompanying smooth vortex contours (Fig. 3 of Ref. 35). In the adjacent larger scales, the superfluid vortices interact with the high enstrophy vortex tubes in the end of the normal-fluid inertial range as discussed in Ref. 37. The latter work showed that the polarization of superfluid turbulence within vortex tubes, first reported in Ref. 42 and analysed further in Refs. 30,43, and 44, is weak compared to the dominant process of spiral-like superfluid vortex formation outside the normal-fluid tubes. The latter process can explain the observed spectral scalings in Fig. 4 as follows: Superfluid vortices become unstable when they interact with intense, normalfluid vorticity regions. Calculations in Refs. 1,37, and 45 show that such instabilities grow to large scales, because of coupling-forces action on the vortices. Both ${ }^{1,37}$ report that the spectral energy scaling corresponding to these instabilities (and the resulting spiral-like vortex structures) is $k^{-3}$. The results of Ref. 1 also indicate that this scaling coexists in $k$ space with the Kolmogorov regime in the normal-fluid. On the other hand, it was shown in Ref. 45, that, during instability growth, the superfluid vortices tend to agglomerate in between the normal-fluid tubes. This result is fully consistent with the nature of the forces acting on the superfluid vortices. In particular, the superfluid vortex model dynamics share basic features with the dynamics of suspensions of inertial particles, i.e., particles that do not follow the flow. Indeed, although inertia can be neglected in the Langevin model, it does not follow that, on time scales of the order of the relaxation time scale $\tau_{R}=\mu_{v} / D_{0}$, the superfluid vortices move with the normal-fluid velocity like passive tracers. This is because, in addition to the mutual-friction force that tends to equalize normal-fluid and vortex velocities, there are also intervortex-Magnus and lift forces on the vortices, as well as, reconnections. The effects of these dynamical factors result in a slip between the normal-fluid velocity and the velocity of the vortices. But in such cases, that is, in cases when suspended particles/vortices slip relative to the fluid, applies a well known result in suspension studies, i.e., that particles tend to accumulate in between the high vorticity regions in the fluid. ${ }^{46}$ This is exactly what happens in finite temperature superfluids too. ${ }^{37,45}$ The agglomeration of superfluid vortices in between the normal-fluid vortex tubes (in areas of low normal-fluid velocity as indicated in Ref. 37) leads to the emergence of weakly 


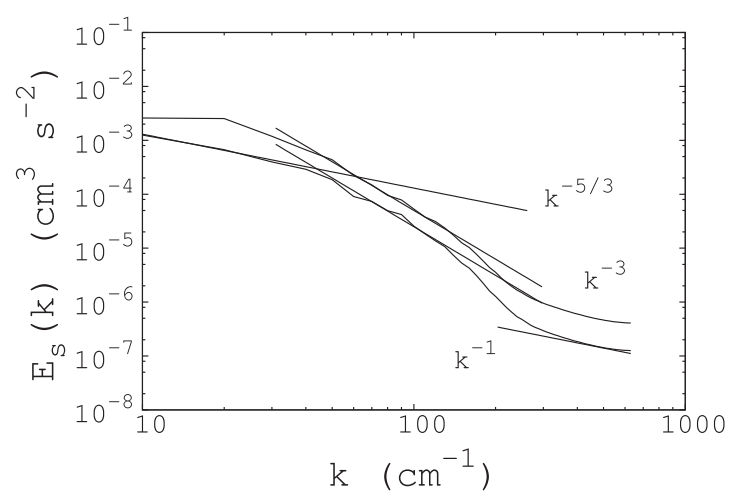

FIG. 4. Superfluid energy spectra at $t=0.0262 \mathrm{~s}$ (bottom curve) and $t=0.0619 \mathrm{~s}$ (top curve). The $t=0.0262 \mathrm{~s} \mathrm{spectrum}$ shows a clear-cut $k^{-5 / 3}$ regime, followed by a steeper $k^{-3}$ range. ${ }^{1,37}$ Both scalings coexist with the Kolmogorov regime in the normal-fluid, and appear well above the intervortex spacing scale. At $t=0.0619 \mathrm{~s}$, the energy levels are too small to support the $k^{-5 / 3}$ regime, and only the $k^{-3}$ is discernible. The corresponding $\left(t-k_{\delta_{i v}}\right)$ pairs are: $(0.0262,293),(0.0619,453)$.

polarized bundles of superfluid vortices. The latter give rise to a Kolmogorov like $k^{-5 / 3}$ regime in the superfluid coexisting with the Kolmogorov range in the normal-fluid. This scaling is clearly indicated in the superfluid energy spectrum at $t=0.0262 \mathrm{~s} \mathrm{(Fig.} \mathrm{4).} \mathrm{The} \mathrm{calculation} \mathrm{shows} \mathrm{that} \mathrm{the}$ $k^{-5 / 3}$ regimes in both fluids vanish at the same time, thus, the normal-fluid inertial range, with its coherent vortex structures ${ }^{45}$ is an essential prerequisite for the emergence of the $k^{-5 / 3}$ regime in the superfluid. In a sense, the Kolmogorov-type spectrum in the superfluid is the result of vortex growth termination, as vortices emanating outwards from different instability centers (which are coherent vortical structures within the normal-fluid's inertial range) collide with each other and agglomerate, in a process reminiscent of caustics formation in particulate suspensions ${ }^{47}$ This also explains why both $k^{-5 / 3}$ and $k^{-3}$ superfluid scalings coexist with the inertial range in the normal-fluid: the coherent structures in the latter are responsible for both the growth of instabilities $\left(k^{-3}\right.$ scaling $)$ and its termination ( $k^{-5 / 3}$ scaling).

Results on the magnitudes of the various forces indicate that the dynamics of the superfluid vortices are dominated by the coupling forces. There can be stretching of the weakly polarized vortexagglomerates in the superfluid, but the magnitude of these effects is orders of magnitude smaller than the magnitude of mutual-friction effects. Indeed, the latter plays the key role in superfluid vortex clustering and the emergence of polarization. Due to its importance, it is worth elaborating upon this point: Consider the standard formulas ${ }^{9}$ for the "potential energy" per unit length $\mathcal{E}(r)$ of two straight, parallel superfluid line vortices, positioned within a cylinder of radius $\delta_{i v} / 2$ and separated by distance $r \gg a_{0}$. In case their vorticities point in the same direction ("parallel vortices"), $\mathcal{E}(r)=\left(\rho_{s} \kappa^{2} / 2 \pi\right) \ln \left(\delta_{i v}^{2} / 4 a_{0} r\right)$, and in case they point in opposite directions ("antiparallel vortices"), and $r \ll \delta_{i v}, \mathcal{E}(r)=\left(\rho_{s} \kappa^{2} / 4 \pi\right) \ln \left(r / a_{0}\right)$. It follows directly that parallel vortices repel each other, i.e., the larger their distance $r$ the smaller the energy of the system, whilst antiparallel vortices attract each other. Evidently, the production (of even weakly) polarized superfluid vorticity bundles ${ }^{45}$ needs external work which is provided here by the mutual-friction and lift-force couplings. Remarkably, in pure superfluid turbulence, without coupling forces, and without the large reservoir of normalfluid kinetic energy, the spontaneous emergence of vortex bundles and associated vortex stretching physics with a Kolmogorov inertial range seems unlikely in the context of this analysis. Notably, this argument does not necessarily exclude a $k^{-5 / 3}$ scaling in a pure superfluid, for the latter can appear for other than vortex-bundle stretching reasons. The $k^{-3}$ regime survives the destruction of the $k^{-5 / 3}$ regime at the later, time $t=0.0619 \mathrm{~s}$, and coexists in $k$ space with the previously mentioned, steep, $k^{-6}$ intermediate scaling in the normal-fluid. Both scalings occur at significantly lower wavenumbers than the intervortex spacing scale wavenumber $k_{\delta_{i v}}=453$. Finally, I have indicated a $k^{-1}$ scaling at high $k$ (Fig. 4). Since the Kelvin waves cascade appears to be damped at $\delta_{i v}$, the $k^{-1}$ spectrum does not correspond to the $k^{-1}$ Kelvin wave cascade scaling derived with dimensional analysis in Ref. 30 and calculated with numerical methods in Ref. 31. Rather, it must correspond to the probing of the 
velocity field of isolated vortex filaments, ${ }^{31}$ especially since it appears below the intervortex space scale wavenumber $k_{\delta_{i v}}$.

\section{EPILOGUE}

The mesoscopic Langevin model proposed here, embeds finite temperature superfluids within the general category of complex fluids. This point of view can have many advantages, leading, for example, to a better understanding of the agglomeration of superfluid vortices in between the normal-fluid vortices, a well known/studied phenomenon in suspensions. More importantly, the mesoscopic model allows the analysis of phenomena not tractable within the framework of standard superfluid vortex dynamics, such as Brownian motion effects or very fast, inertial, vortex relaxation processes. Advanced numerical methods for complex fluids in general, and Langevin equations in particular, could also eventually be applied to superfluids.

The interplay of numerics with physics is the major concern in the proposed methodology for the design of well resolved numerical calculations of finite temperature superfluids. It relies on the crucial observation of apparent Kelvin wave cascade damping in fully coupled normalfluid/superfluid calculations. Therefore, a better resolved (perhaps also with more accurate numerical methods) computation of the flow in Ref. 35 is a desirable immediate development. New advances in numerical vortex dynamics ${ }^{48}$ can also inform computational work in superfluids, especially in the context of the systematic desingularization of the superfluid-vortex Biot-Savart integral.

Regarding the fecund spectral structure of finite temperature superfluids, the computed low $k, k^{-5 / 3}$ spectra in both fluids are consistent with previous experimental conclusions. ${ }^{49,50}$ In this context, it would be highly desirable if the metastable Helium molecules technique of Ref. 51 could evolve to the point of resolving and measuring actual normal-fluid spectra. This would be a true major step ahead in superfluid hydrodynamics research. Another key development would be the design of massively parallel algorithms that could push to higher values the Reynolds number in the normal-fluid and vortex tangle densities in the superfluid. Moreover, by increasing the numerical resolution along the vortices, it will be possible to directly investigate the damping of the Kelvin wave cascade within a fully developed turbulence computation, rather than referring to the specialized computation results of Ref. 35. The implementation of computational operators that educe superfluid vortex polarization in the $k^{-5 / 3}$ energy scaling regime of a complex vortex tangle is also of central importance. Approaches based on Minkowski functionals, ${ }^{52}$ for example, can be very suitable for this purpose.

\section{ACKNOWLEDGMENTS}

I am grateful to Andrei Golov for indicating to me the bottom-up approach to vortex dynamics of Thompson and Stamp, ${ }^{16}$ and especially to Joe Vinen for numerous discussions on the nature of the quasiparticle forces on superfluid vortices.

${ }^{1}$ D. Kivotides, "Spreading of superfluid vorticity clouds in normal-fluid turbulence," J. Fluid Mech. 668, 58 (2011).

${ }^{2}$ R. J. Donnelly, Quantized Vortices in Helium II (Cambridge University Press, 1991).

${ }^{3}$ W. F. Vinen and J. J. Niemela, "Quantum turbulence,” J. Low Temp. Phys. 128, 167 (2002).

${ }^{4}$ S. K. Nemirovskii, "Quantum turbulence: Theoretical and numerical problems," Phys. Rep. 524, 85 (2013).

${ }^{5}$ P. M. Walmsley and A. I. Golov, "Quantum and quasiclassical types of superfluid turbulence," Phys. Rev. Lett. 100, 245301 (2008).

${ }^{6}$ A. P. Finne, V. B. Eltsov, R. Hanninen, N. B. Kopnin, J. Kopu, M. Krusius, M. Tsubota, and G. E. Volovik, "Dynamics of vortices and interfaces in superfluid ${ }^{3} \mathrm{He}$, , Rep. Prog. Phys. 69, 3157 (2006).

${ }^{7}$ S. N. Fisher and G. R. Pickett, "Quantum turbulence in superfluid ${ }^{3} \mathrm{He}$ at very low temperatures," Prog. Low Temp. Phys. 16, 147 (2009).

${ }^{8}$ K. W. Schwarz, "Three-dimensional vortex dynamics in superfluid ${ }^{4} \mathrm{He}$ : Line-line and line-boundary interactions," Phys. Rev. B 31, 5782 (1985).

${ }^{9}$ A. J. Leggett, Quantum Liquids (Oxford University Press, 2006).

${ }^{10}$ J. Yepez, G. Vahala, M. Vahala, and M. Soe, "Superfluid turbulence from quantum Kelvin wave to classical Kolmogorov cascades," Phys. Rev. Lett. 103, 084501 (2009).

${ }^{11}$ M. Tsubota and M. Kobayashi, "Energy spectra of quantum turbulence," Prog. Low Temp. Phys. 16, 1 (2009). 
${ }^{12}$ D. Jou, M. S. Mongiovi, and M. Sciacca, "Hydrodynamic equations of anisotropic, polarized and inhomogeneous superfluid vortex tangles," Physica D 240, 249 (2011).

${ }^{13}$ G. Baym and E. Chandler, "Hydrodynamics of rotating superfluids, I. Zero temperature, nondissipative theory," J. Low Temp. Phys. 50, 57 (1983).

${ }^{14}$ M. Rubinstein and R. H. Colby, Polymer Physics (Oxford University Press, 2003).

${ }^{15}$ C. E. Brennen, Fundamentals of Multiphase Flow (Cambridge University Press, 2005).

${ }^{16}$ L. Thompson and P. C. E. Stamp, "Quantum dynamics of a Bose superfluid vortex," Phys. Rev. Lett. 108, 184501 (2012)

${ }^{17}$ B. Andreotti, Y. Forterre, and O. Pouliquen, Granular Media (Cambridge University Press, 2013).

${ }^{18}$ E. B. Sonin, "Magnus force in superfluids and superconductors," Phys. Rev. B 55, 485 (1997).

${ }^{19}$ E. A. Calzetta and B. B. Hu, Nonequilibrium Quantum Field Theory (Cambridge University Press, 2008).

${ }^{20}$ S. K. Nemirovskii, "Thermodynamic equilibrium in the system of chaotic quantized vortices in a weakly imperfect Bose gas," Theor. Math. Phys. 141, 1452 (2004).

${ }^{21}$ T. Zhang and S. W. Van Sciver, "The motion of micron-sized particles in HeII counterflow as observed by the PIV technique," J. Low Temp. Phys. 138, 865 (2005).

${ }^{22}$ D. Kivotides, "Normal-fluid velocity measurement and superfluid vortex detection in thermal counterflow turbulence," Phys. Rev. B 78, 224501 (2008).

${ }^{23}$ D. Kivotides, S. L. Wilkin, and T. G. Theofanous, "Stochastic entangled chain dynamics of dense polymer solutions," J. Chem. Phys. 133, 144903 (2010).

${ }^{24}$ D. Kivotides, Y. A. Sergeev, and C. F. Barenghi, "Dynamics of solid particles in a tangle of superfluid vortices at low temperatures," Phys. Fluids 20, 055105 (2008).

${ }^{25}$ A. W. Baggaley, "The sensitivity of the vortex filament method to different reconnection models," J. Low Temp. Phys. 168, 18 (2012).

${ }^{26}$ T. S. Lundgren, "Linearly forced isotropic turbulence," Annual Research Briefs (Stanford Center for Turbulence Research, 2003), p. 461.

${ }^{27}$ C. Rosales and C. Meneveau, "Linear forcing in numerical simulations of isotropic turbulence: Physical space implementations and convergence properties," Phys. Fluids 17, 095106 (2005).

${ }^{28}$ P. L. Carroll and G. Blanquart, "A proposed modification to Lundgren's physical space velocity forcing method for isotropic turbulence," Phys. Fluids 25, 105114 (2013).

${ }^{29}$ B. V. Svistunov, "Superfluid turbulence in the low-temperature limit," Phys. Rev. B 52, 3647 (1995).

${ }^{30}$ W. F. Vinen, "Classical character of turbulence in quantum liquid," Phys. Rev. B 61, 1410 (2000).

${ }^{31}$ D. Kivotides, J. C. Vassilicos, D. C. Samuels, and C. F. Barenghi, "Kelvin waves cascade in superfluid turbulence," Phys. Rev. Lett. 86, 3080 (2001).

${ }^{32}$ E. Kozik and B. V. Svistunov, "Kelvin-wave cascade and decay of superfluid turbulence," Phys. Rev. Lett. 92, 035301 (2004).

${ }^{33}$ V. S. L'Vov and S. Nazarenko, "Spectrum of Kelvin-wave turbulence in superfluids," JETP Lett. 91, 428-434 (2010).

${ }^{34}$ W. F. Vinen, "Decay of superfluid turbulence at a very low temperature: The radiation of sound from a Kelvin wave on a quantized vortex," Phys. Rev. B 64, 134520 (2001).

${ }^{35}$ D. Kivotides, "Turbulence without inertia in thermally excited superfluids," Phys. Lett. A 341, 193 (2005).

${ }^{36}$ D. Kivotides, "Relaxation of superfluid vortex bundles via energy transfer to the normal fluid," Phys. Rev. B 76, 054503 (2007).

${ }^{37}$ D. Kivotides and S. L. Wilkin, "Elementary vortex processes in thermal superfluid turbulence," J. Low Temp. Phys. 156, 163 (2009).

${ }^{38}$ O. C. Idowu, D. Kivotides, C. F. Barenghi, and D. C. Samuels, "Equation for self-consistent superfluid vortex line dynamics," J. Low Temp. Phys. 120, 269 (2000).

${ }^{39}$ D. Kivotides, S. L. Wilkin, and T. G. Theofanous, "Stretching of polymer chains by fluctuating flow fields," Phys. Lett. A 375, 48-52 (2010).

${ }^{40}$ D. Kivotides, C. F. Barenghi, and D. C. Samuels, “Triple vortex ring structure in superfluid helium II," Science 290, 777 (2000).

${ }^{41}$ D. Kivotides, D. C. Samuels, and C. F. Barenghi, "Fractal dimension of superfluid turbulence," Phys. Rev. Lett. 87, 155301 (2001).

${ }^{42}$ D. C. Samuels, "Response of superfluid vortex filaments to concentrated normal-fluid vorticity," Phys. Rev. B 47, 1107 (1993).

${ }^{43}$ C. F. Barenghi, S. Hulton, and D. C. Samuels, "Polarisation of superfluid turbulence," Phys. Rev. Lett. 89, 275301 (2002).

${ }^{44}$ K. Morris, J. Koplik, and D. W. Rousson, "Vortex locking in direct numerical simulations of quantum turbulence," Phys. Rev. Lett. 101, 015301 (2008).

${ }^{45}$ D. Kivotides, "Coherent structure formation in turbulent thermal superfluids," Phys. Rev. Lett. 96, 175301 (2006).

${ }^{46}$ K. D. Squires and J. K. Eaton, "Particle response and turbulence modification in isotropic turbulence," Phys. Fluids A2, 1191 (1990).

${ }^{47}$ M. W. Reeks, "Transport, mixing and agglomeration of particles in turbulent flows," Flow Turbul. Combust. 92, 3 (2014).

${ }^{48}$ A. Leonard, "On the motion of thin vortex tubes," Theor. Comput. Fluid Dyn. 24, 369 (2010).

${ }^{49}$ J. Maurer and P. Tabeling, "Local investigation of superfluid turbulence," Europhys. Lett. 43, 29 (1998).

${ }^{50}$ S. R. Stalp, L. Skrbek, and R. J. Donnelly, "Decay of grid turbulence in a finite channel," Phys. Rev. Lett. 82, 4831 (1999).

${ }^{51}$ W. Guo, J. D. Wright, S. B. Cahn, J. A. Nikkel, and D. N. McKinsey, "Metastable Helium molecules as tracers in superfluid ${ }^{4}$ He," Phys. Rev. Lett. 102, 235301 (2009).

${ }^{52}$ S. L. Wilkin, C. F. Barenghi, and A. Shukurov, "Magnetic structures produced by the small-scale dynamo," Phys. Rev. Lett. 99, 134501 (2007) 